\title{
Robotics and Virtual Reality Applications in Mobility Rehabilitation
}

\author{
Rares F. Boian ${ }^{1}$, Grigore C. Burdea ${ }^{2} \&$ Judith E. Deutsch ${ }^{3}$ \\ ${ }^{1}$ Department of Mathematics and Computer Science, Babeş-Bolyai University, Romania \\ ${ }^{2}$ CAIP Center, Rutgers University, New Jersey, USA \\ ${ }^{3}$ Rivers Lab SHRP, University of Medicine and Dentistry, New Jersey, USA
}

\section{Introduction}

Gait training is a method to reduce mobility dysfunction. Diverse patient populations exhibit mobility impairments that can be ameliorated with gait training. Two such populations are people post-stroke and post-spinal cord injury.

The ability to walk is one of several functions affected by stroke. Immediately after the stroke only $37 \%$ of the survivors are able to walk (Jorgensen et al., 1995). Of the patients with initial paralysis only $21 \%$ regain walking function (Wandel et al., 2000).

Another patient population that can benefit from gait training (Dietz et al., 1998; Nicol et al., 1995) is the spinal cord injury victims. According to the Travis Roy Foundation there are currently between 250,000 and 400,000 Americans living with spinal cord injury.

Gait training or locomotion therapy uses several devices to assist the patient move and maintain balance. Canes, crutches, walkers, and platforms are simple ambulatory assistive $\varepsilon$ devices that modify a patient's independence and functional mobility. Treadmills often ठ equipped with un-weighing devices are used for training walking at various speeds on a $\Phi$ straight flat surface or small incline. These features, along with treadmills' simple design 言 and affordable costs are sufficient reasons for their popularity. Treadmills, however, cannot 들 render more complex walking surfaces which patients encounter daily, such as: stairs, of curves, uneven surfaces (e.g. cobblestone paths), or surfaces with various stiffness or friction $\stackrel{ \pm}{.}$ coefficients.

Training patients to negotiate complex walking surfaces can be done either through in-vivo training assisted by a physical therapist, or through using devices able to simulate such $£$ surfaces. The former approach often takes the patient out of the controlled clinic If environment, which is not always feasible and may raise safety concerns. The latter 즐 approach may offer an alternative to real environment training. It would allow patients to त्ञ exercise in controlled and safe conditions in the clinic, which could potentially be more time of and personnel efficient than real environment training, In this context, numerous research \& projects have approached gait simulators trying to create robotic devices that could render ¿্ৰ complex walking surfaces.

The integration of such robotic systems with virtual environments may, in theory, expand Фे the range of applications to entertainment and real-life task training of patients with $\bigcirc$ walking dysfunction. The appealing reasons for using such systems are the flexibility and 
transparent data collection offered by virtual environments over real environments (Iwata, 1999; Sveistrup, 2004). In addition, the ability to train for a task in a controlled environment, away from the potential hazards of a real environment makes such simulators viable choices in medical or military applications.

\section{Locomotion Simulators}

Locomotion simulators, which attempt to simulate the sensations of walking, have been the focus of many researchers due to their applicability in simulating real-life tasks. Beside the medical reasons presented above, gait simulators are an attractive subject due to their applicability for military training or gaming. However, the design of a locomotion simulator for therapeutic purposes must consider and possibly solve several aspects raised by the people using it and by the environment where it will be used.

- $\quad$ Safety - The first and most important aspect is safety. Gait simulators are usually complex robotic devices on which the patient stands and moves. To avoid accidents, the simulator must be constrained to move only within the physiological limits of the human body. It also must provide the patient with means to quickly reach safety should anything wrong happen with the simulator.

- Environment - A gait simulator must also be suitable for usage in a clinic or home environment. Given the size, these devices are most often used in clinics, but there are research projects (the simulator presented in the last part of this chapter included) that aim to reduce the simulator's size. The environment also imposes restrictions on the actuators. Hydraulic actuators are appropriate for balancing the weight of a person, but they are unsuitable for medical usage, because they are impossible to keep clean, and also pose the risk of dangerous leakages.

- Interference with patient - Although not always possible, a simulator should allow the patient to move freely, without constraining him or her. This implies supporting normal step lengths, various locomotion speeds, and changes of direction. Solutions for this requirement usually impose compromises on the size of the simulator.

- Mechanical bandwidth - The human haptic sensory capabilities require a force display to rendering bandwidth of about $1 \mathrm{KHz}$ (Burdea, 1996), while the human motor actions require around $10 \mathrm{~Hz}$ bandwidth. Thus, a walking simulator needs to render forces at $10 \mathrm{~Hz}$ to be able to follow the patient's motion. In order to simulate more complex walking surfaces, forces should ideally be applied at $1 \mathrm{KHz}$ bandwidth. However, these forces are usually felt through shoes, so there is no need of such high fidelity.

- Surface simulation - The interaction between a gait simulator and the patient is defined by the surface to be rendered. In order to render complex surfaces realistically, the contact between the simulator and the patient's foot should include multiple active points that define the shape of the surface. Ideally, the simulator should also support changes of walking direction and surface inclination. The solutions to all these issues depend primarily on the design and mechanical limits of the simulator. 
- Assistive mode - The design of walking simulators for physical therapy also needs to consider assistive mode functioning. As the patients are likely to have difficulty walking, it can be useful to actively guide their feet while walking.

- Data collection - One benefit of involving robotics in the rehabilitation procedures is the possibility of collecting data about the patient actions and motions. Data measured during exercises can then be processed and serve as objective base for progress evaluation.

The design of a gait simulator poses numerous issues to be solved, besides those listed above. However, all designs must address one essential requirement: to create the sensation of walking on an infinite surface. The treadmill design solves this problem with a straight forward approach, but is limited to simulating an infinite straight smooth path. To rendered surfaces richer in features, researchers devised several designs, which Hollerbach (Hollerbach, 1999) classifies into three categories: walk-in-place devices, treadmills, and foot platforms.

\subsection{Walk-in-Place Devices}

These devices require the user to walk in place without advancing while his or her motions are tracked by sensors. The recorded data are then interpreted by a driving workstation that computes the direction and speed of the virtual avatar and changes the view in the virtual environment. These systems do not output any haptic feedback to the user. The only forces the user feels is the contact with the floor.

Templeman et al (Templeman et al, 1999) and Parsons et al. (Parsons et al., 1998) developed such systems using magnetic trackers to measure the user's motion and infer the direction and speed of walking.

Iwata tried the same approach using slippery shoes and asked the user to walk normally (Iwata \& Yoshida, 1999). An improved walk-in-place device is presented in Bouguilla et al. (Bouguilla \& Sato, 2002). The user walked on a turntable that counteracted the user's change of direction by rotating in the opposed direction.

Compared to a regular treadmill, the walk-in-place systems bring the possibility of changing the walking direction but do not allow the patients to actually walk with normal gait. Iwata's approach with slippery shoes may be risky when dealing with people with disabilities.

\subsection{Treadmills}

The treadmill category includes devices that allow the user to walk normally on top of a mobile surface that slides in the direction opposed to that of walking.

Such a treadmill is the Sarcos Treadport (Christensen et al., 2000) which can simulate steep up-hill walking and inertial forces.

The Torus treadmill (Iwata, 1999) allowed the user to walk in any direction at a maximum speed of $0.5 \mathrm{~m} / \mathrm{sec}$.

Another omni-directional treadmill is presented in (Wang et al., 2003). The device developed by Wang et al. used a low friction cloth on top of a rigid board. The cloth was moved in the direction opposite to that of walking by high-friction casters pressed against the board.

The ATR-GSS device presented in (Miyasato, 2000) is a regular treadmill instrumented with mobile plates under the belt. Various walking surface shapes can be simulated by moving the plates up and down. 
The advantages brought by these systems when compared with regular treadmills are readily apparent. The change in direction and up-hill walking are frequent daily life scenarios, for which the patients could be trained for. The disadvantage of these devices is that they were not designed for physical rehabilitation, thus their deployment in a clinic is problematic due to size and actuator choices.

The Lokomat System and the Robomedica BWS System are walking simulators developed specifically for clinical usage. Both of them rely on a regular treadmill and provide an actuated systems for dynamically supporting the patient's weight. The Lokomat system, designed for paraplegic rehabilitation, also features an exoskeleton that can guide the patient's legs through the normal gait cycle.

\subsection{Foot Platforms}

This category includes devices that use one actuated platform for each foot. Depending on the degrees of freedom of the platforms, these systems may be able to simulate complex surfaces, by controlling the position and orientation of the surface at each foot.

Examples of foot platforms are the Sarcos Biport (Hollerbach, 1999) that uses 2DOF platforms and the GaitMaster (Iwata et al., 2001) that uses 3DOF platforms. Just like the treadmills above, the foot platform system presented in this section feature flexibility for rendering more complex surfaces, but their design was not meant for clinical usage.

\subsection{Other Walking Simulators}

A very realistic simulation of uneven terrain is the Terrain Surface Simulator ALF presented in (Noma et al. 2000). The simulator is a rectangular surface made of small tilt-able plates that can be controlled in real-time. By changing the orientation of the plates, the walking surface can be set in a large variety of shapes. This device is not a treadmill so the user can only walk in any direction within the actuated area.

E-motek Inc. (Amsterdam, Netherlands) has developed the CAREN system, a hydraulically actuated Stewart platform robot supporting a 2-meter diameter board for simulating surfaces with any tilt angle.

VirtuSphere Inc. (Redmond, WA) develops a virtual sphere large enough for a person to fit in. The sphere is made of low-friction material and is supported by a system of casters that allow it to rotate as the user walks.

\section{Virtual Environments}

The integration of gait simulators with virtual environments makes possible task specific training in the clinic. A patient immersed in a virtual world while exercising may find the therapy less tedious and may also be more motivated (Riva, 2000).

A gait simulator integrated with a VR simulation has to accomplish two main tasks:

map the user's motion into virtual environment navigation and calculate the haptic feedback to be applied to the user's feet or legs as a result of the interactions in the virtual world. The extent to which these tasks are implemented depends mainly on the limitations imposed by the simulator system.

In most situations, the applicability and success of a virtual reality simulation is conditioned by the degree of video and audio feedback. For physical-based simulations, the realism is 
also defined by how close the haptic feedback feels compared to the real life experience. In the case of gait simulators, the haptic feedback is calculated from the interaction between a virtual avatar and a virtual surface and applied primarily to the feet.

\section{Dual Stewart Platform Mobility Simulator}

The main hardware components of the Mobility Simulator are two 6DOF prototype Rutgers Mega-Ankle (RMA) pneumatic robots (see Figure 1). Each robot has a Stewart platform configuration with six dual-acting pneumatic pistons. Each piston is mounted in parallel with a linear potentiometer, which provides information of the smaller mobile platform position vs. the larger fixed one. The mobile platform has a foot attachment plate and a $6 \mathrm{DOF}$ force sensor. Force data are used when the Rutgers Mega-Ankle robot is in force control mode. The Mobility Simulator also incorporates an electro-pneumatic control interface, an un-weighting frame (Biodex Co.), a graphical workstation and a large screen custom display. The design of the simulator fits in the "foot platforms" category defined by Hollerbach (Hollerbach, 1999). The user stands with each foot secured with straps to the top of a platform, while the VR simulation (Boian et al., 2004b) is displayed on the large screen facing the user. To improve performance and safety, the user's body is strapped in the unweighting frame placed above the two platforms. For lightweight users unloading is not necessary, safety being provided by the handlebars mounted on the frame's posts. In this setup, the user can walk on top of the RMA platforms with small steps limited by the platform's workspace (Boian et al., 2003).

During walking, each foot either supports the weight of the body or swings freely while taking a new step. Accordingly, each RMA platform can function in load compensation mode or in free motion mode. In free motion mode the RMA platform follows the swinging foot and compensates for its own mobile platform weight. During this mode, the platform applies very low to zero forces to the foot. In load compensation mode, the platform holds the supporting foot weight and slides backward simulating the behavior of a treadmill. In both modes, the robots can apply additional 6DOF forces or vibrations to the user's foot as commanded by the simulation running on the workstation. The two functioning modes mentioned above are a subset of the actual implementation, sufficient for the purpose of this paper. A more detailed description of the RMA platform's functioning modes can be found in (Boian, 2005).

The simulator software is distributed on two computers: the graphics workstation and the electro-pneumatic control interface (an embedded PC) as illustrated in Figure 2. The graphics workstation handles the graphic and haptic rendering. Based on the information received from the control interface, it calculates the interaction between the virtual feet and the virtual environment and sends back to the control interface commands regarding the forces to be applied or the functioning mode to be used.

The control interface handles the low-level servo control of the two robots, and provides the simulator with the position of each foot calculated through forward kinematics. For performance purposes, the control interface also takes care of switching between certain functioning modes based on the forces applied by the foot as measured by the Rutgers Mega-Ankle 6DOF force sensor. The current functioning mode of each RMA platform is also sent to the graphics workstation. A more detailed presentation of the tasks executed by the control interface can be found in (Boian et al. 2003). 


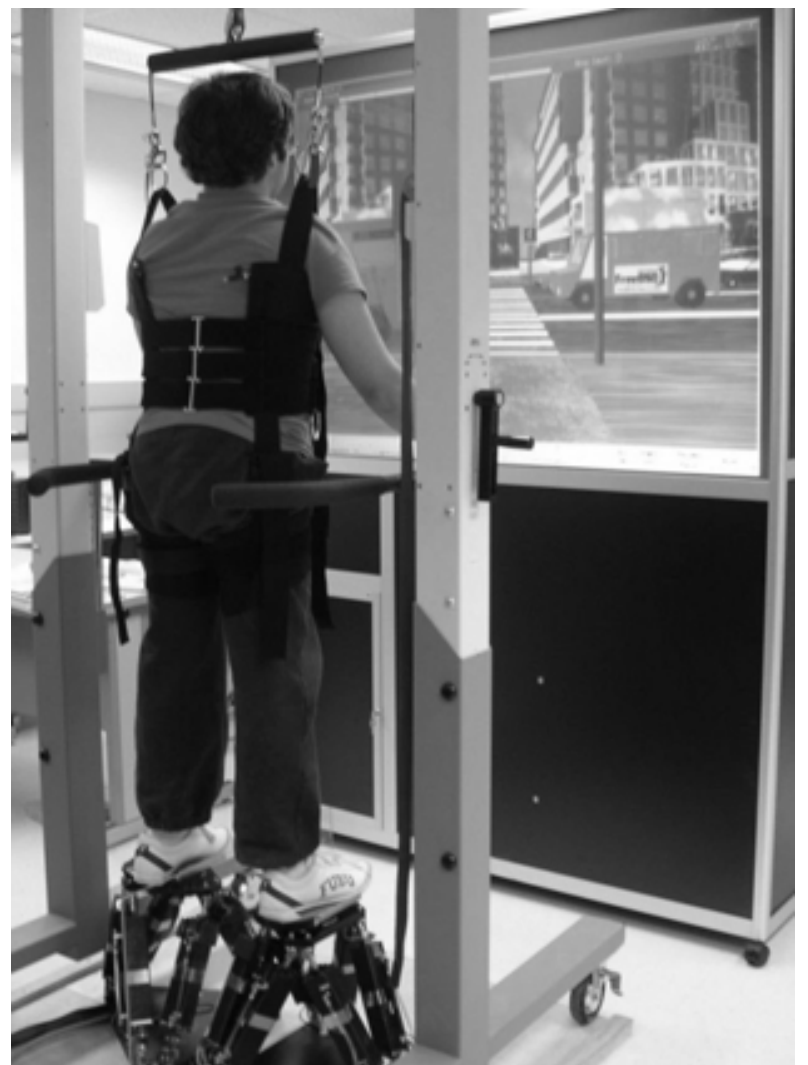

Fig. 1. The Rutgers Mobility Simulator. (C) Rutgers University and UMDNJ. Reprinted by permission.

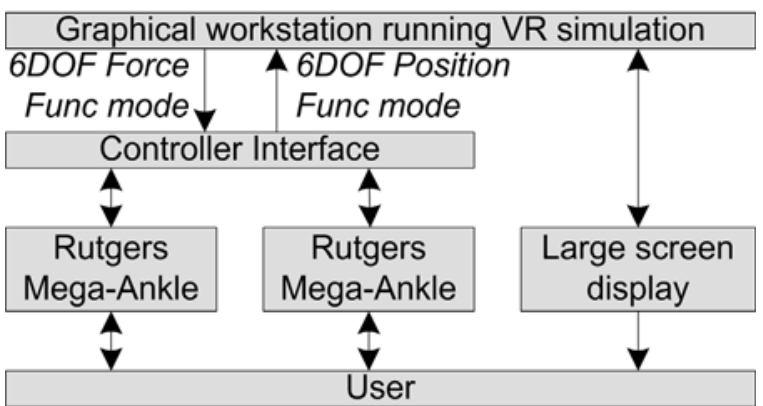

Fig. 2. The Mobility Simulator connection diagram.

\subsection{The Rutgers Mega-Ankle servo control}

The servo controller design is split over three loops represented as shaded areas in Figure 3. From top to bottom, the loops are: task control loop, dynamics loop, and pressure loop. 


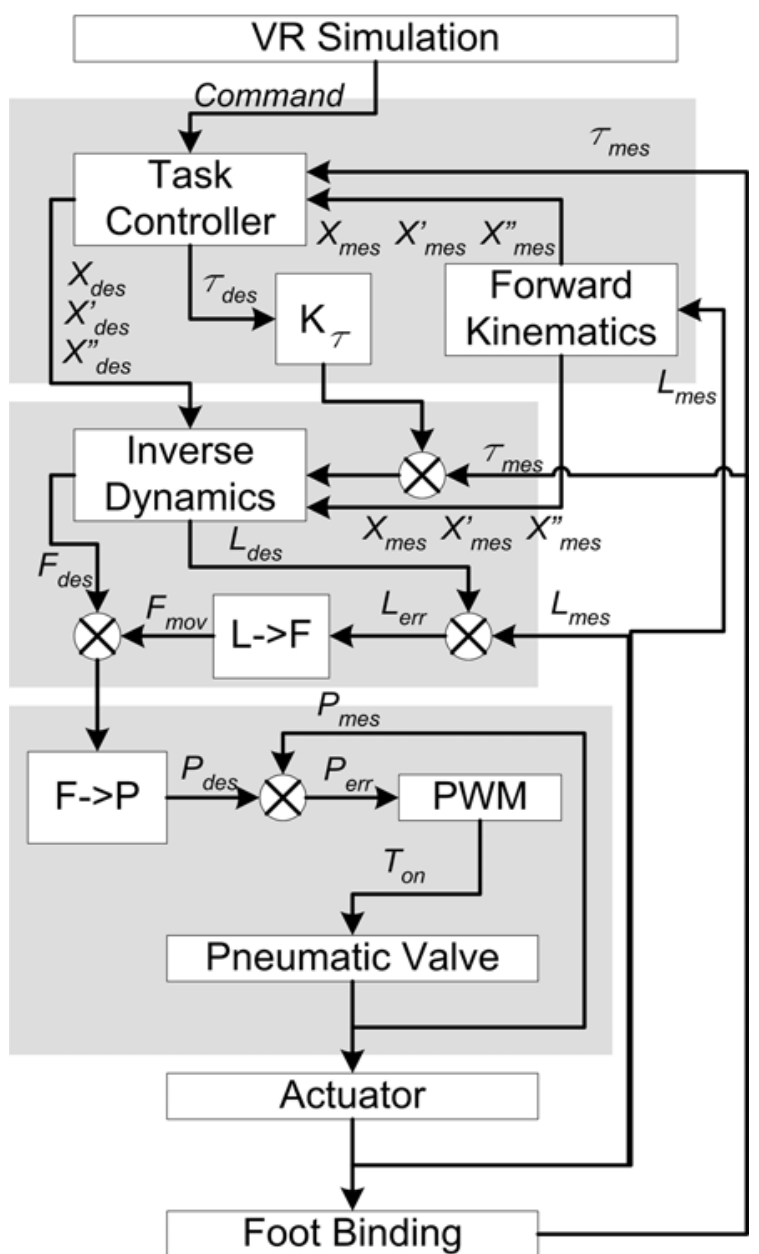

Fig. 3. Controller architecture. (C) Rutgers University and UMDNJ. Reprinted by permission.

The task control loop is responsible for processing the commands coming from the VR simulation and converting them into Stewart platform desired position, velocity, acceleration and forces. The commands coming from the simulation specify the functioning mode to be used (i.e. weight support mode or free motion mode), and haptic effects and forces to be applied to the users feet. This loop is executed alternatively for each platform. The task controller converts the simulation command using as additional input the measured positions and forces, read by the linear potentiometers and the force sensor of each robot. The dynamics loop transforms the desired Cartesian positions and forces into actuator level forces. The inverse dynamics implementation takes into account the current state of the robot, including position, velocity, acceleration and external forces. The measure external forces $\mathrm{T}_{\text {mes }}$ are added with the desired forces $\mathrm{T}_{\text {des }}$ calculated by the task control loop, hence the final force being applied will be the force necessary to counteract the user's action added with the desired force to be applied to the user's foot. 
The actuators used by the RMA robots are dual-acting pneumatic pistons, hence the force applied by the cylinder can by controlled by adjusting the air pressure in the upper and lower chambers. The actuator position control transforms the desired change in position into a desired force. The calculated cylinder lengths $\mathrm{L}_{\mathrm{des}}$ are added with the measured length $\mathrm{L}_{\text {mes, }}$ and the resulting length difference Lerr is transformed into a force through the $\mathrm{L} \rightarrow \mathrm{F}$ control block. This control uses a proportional derivative (PD) strategy.

Finally, the pressure loop takes the desired actuator force, transforms it into upper and lower chamber pressures. The desired pressures are controlled in Pulse Width Modulation (PWM). The PWM strategy transforms the desired change in pressure $P_{\text {err }}$ into the time interval $\mathrm{T}_{\text {on }}$ during which the valve should be kept open.

$\mathrm{F} \rightarrow \mathrm{P}$ is a direct transformation of actuator force into upper and lower pressures designed to minimize the change in pressures in both chambers, hence achieving a better response time. In cases when the desired force cannot be achieved through minimization, the pressures are determined so that they are balanced around the middle of the controllable pressure range. The PWM is implemented using an adaptive strategy. The change in pressure in a cylinder chamber is not linear in time, and the shape of the curve depends on the volume of the actuated cylinder chamber. Another factor taken into consideration is the intake airflow, which is affected by the number of actuator chambers accepting or exhausting air simultaneously. The PWM duty cycle is calculated taken into consideration all these factors using the equation below.

$$
T_{\text {on }}=k P_{\text {err }}\left(1+k_{V} V_{\text {air }}\right)\left(1+k_{N} N_{\text {chamber }}\right)\left(1+k_{F} F_{\text {flow }}\right)
$$

$\mathrm{V}_{\text {air }}$ is the volume of uncompressed air necessary to achieve the change in pressure. $\mathrm{N}_{\text {chamber }}$ is the number of chambers accepting or exhausting air simultaneously. $\mathrm{F}_{\text {flow }}$ is the curve of the airflow over time.

\subsubsection{Simulator Mechanical Bandwidth}

The mechanical bandwidth of the mobility simulator was measured for translations in the horizontal plane and rotations around the front/back axis. The motions are the most commonly used by our system to render haptic effects as discussed in the last section of the paper. The measurements were done with both RMA robots active simultaneously. While the bandwidth of each individual robot is higher when measured separately, when both robots are active the intake airflow is reduced, thus the bandwidth is lowered. The results are presented in Table 1.

\begin{tabular}{|c|c|c|}
\hline $\begin{array}{c}\text { X translation } \\
0.1 \mathrm{~m} \text { amplitude }\end{array}$ & $\begin{array}{c}\text { Y translation } \\
0.18 \mathrm{~m} \text { amplitude }\end{array}$ & $\begin{array}{c}\text { Y rotation } \\
5 \mathrm{deg} \text { amplitude }\end{array}$ \\
\hline $1.56 \mathrm{~Hz}$ & $1.79 \mathrm{~Hz}$ & $1.5 \mathrm{~Hz}$ \\
\hline
\end{tabular}

Table 1. RMA robot mechanical bandwidth.

\subsubsection{Robot Stability in Foot Support Mode}

One of the first problems encountered during the development of the system was the stability of the RMA platforms under load. The robots were stable when subjected to external forces if there was no load attached to them. However, the working regime for which they were developed, involves resisting forces while supporting the weight of the user. Figure 4 shows the response of the robot to sinusoidal input. Under a $50 \mathrm{lbs}$ load, the 
motion was distorted and the amplitude of the robot increased slowly eventually becoming unstable.

The cause of this problem was the addition of the desired cylinder force $F_{\text {des }}$ with the cylinder motion force $\mathrm{F}_{\text {mov. }}$. Under load, the resulting forces were too high and caused the robot to become unstable. Increasing the derivative gain slowed down the instability but it didn't solve it. The solution was to reduce the proportional gains by a minimum of $42 \%$. With lower gains, the platform was stable under load, but had very little power to move the user's foot backward during the foot support phase, hence making the system unusable. Also, when the system was unloaded, the steady state error was significantly larger. The use of an integrator term was avoided because the usage of the system caused it to windup consistently.

Two adaptive gains were used to bring the robot to respond properly under load. The gains added a fraction of the measured cylinder load to the proportional and derivative gains respectively. The proportional adaptive component helped increase the moving force of the platform when under load, while the derivative adaptive component was increased to compensate for the high proportional gains and insure the stability of the system.

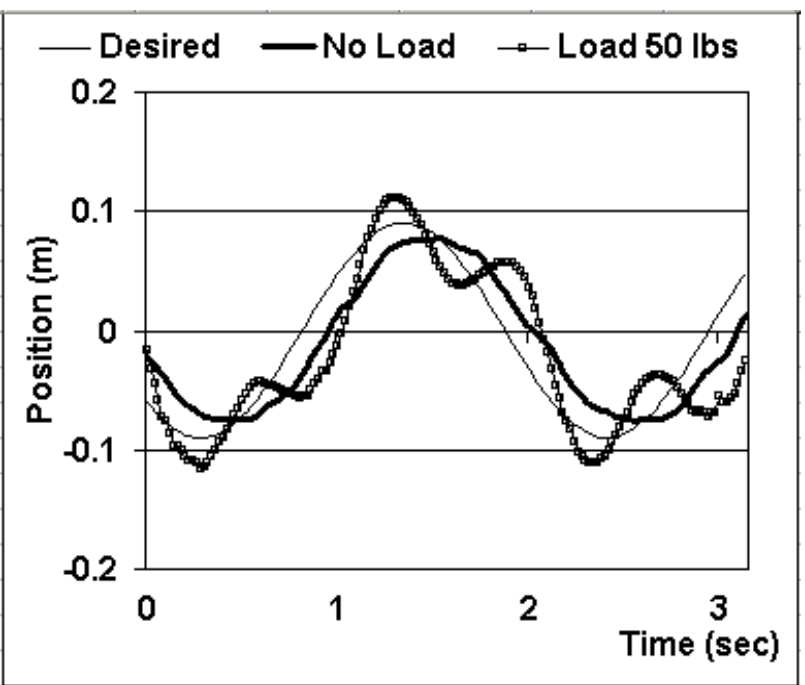

Fig. 4. RMA platform response to a sinusoid input along the $\mathrm{Y}$ axis (back-front) with $0.5 \mathrm{~Hz}$ frequency and $0.18 \mathrm{~m}$ amplitude. (C) Rutgers University and UMDNJ. Reprinted by permission.

Figure 5 presents the response of the platform to the same sinusoidal input signal, under a $50 \mathrm{lbs}$ load, with and without the adaptive component added to the lowered proportional and derivative gains. While both the constant and the adaptive response were stable, the adaptive strategy provided the necessary power to move the load closer to the desired position, and reducing the error by approximately half. The adaptive gains did bring a side effect slightly visible in Figure 5; at higher velocity, the adaptive derivative gains increases the damping of the system slowing it down and the releasing it as the load $n$ the robot shifts and the force is reduced. This can be seen as a change in the slope of the adaptive curve in Figure 5. 


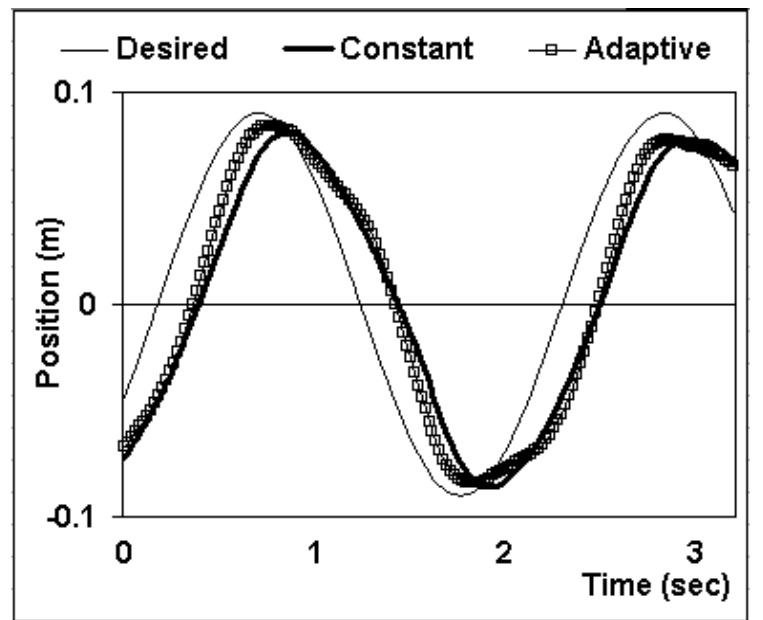

Fig. 5. Response comparison lower constant gains with and without the adaptive component. (C) Rutgers University and UMDNJ. Reprinted by permission.

\subsubsection{Force Minimization in Free Motion Mode.}

The second major functioning mode of the RMA robot is following the foot during the swing phase of the gait. In this mode, the platform has to compensate for its own weight and for the forces applied by the user to the end-effector, hence not making itself felt to the foot. To achieve this, the servo controller disables the cylinder position control by canceling out the moving force $F_{\text {mov, }}$ and by switching the measured forces signs by changing the value of $K_{t}$ from 1 to -1 . While these changes cause the RMA robot to follow the user's foot, the motion is very slow and large forces are felt at the foot. Figure 6 shows the forces measured at the foot during one swing phase.

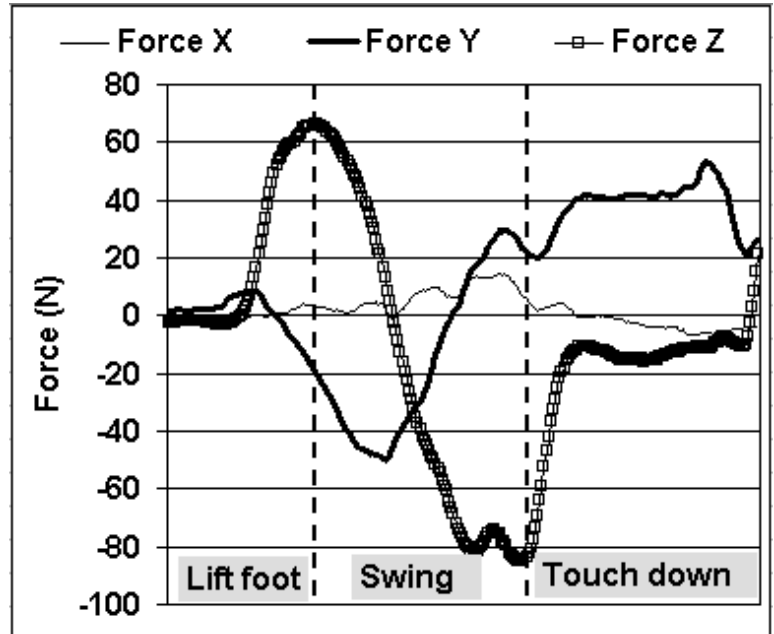

Fig. 6. Free mode forces during swing for $K_{t}=-1$. 
The source of these large forces has been determined to be the damping created by the pneumatic actuators. While a $K_{t}$ of 1 is sufficient to resist forces in foot support mode, in free mode, besides responding with a force to the user's force, the robot also has to move, which involves a much large air intake/exhaust activity. The damping is mostly coming from the airflow limitations imposed by the small intake and exhaust sections of the cylinder chambers. To overcome this problem, $\mathrm{K}_{\mathrm{t}}$ was increased in absolute value. The increase was done for each of the robot's 6 DOF. The Z-axis (up-down) translation gain was approximately four times larger than the rest of the gains, because the motion on that direction required all the cylinders to either intake or exhaust, hence putting more airflow in a single direction. The measured forces for the increased $K_{t}$ gains are shown in Figure 7 . The forces are now reduced approximately 8 times to a maximum of $11 \mathrm{~N}$, which is comparable to the weight of a snow boot.

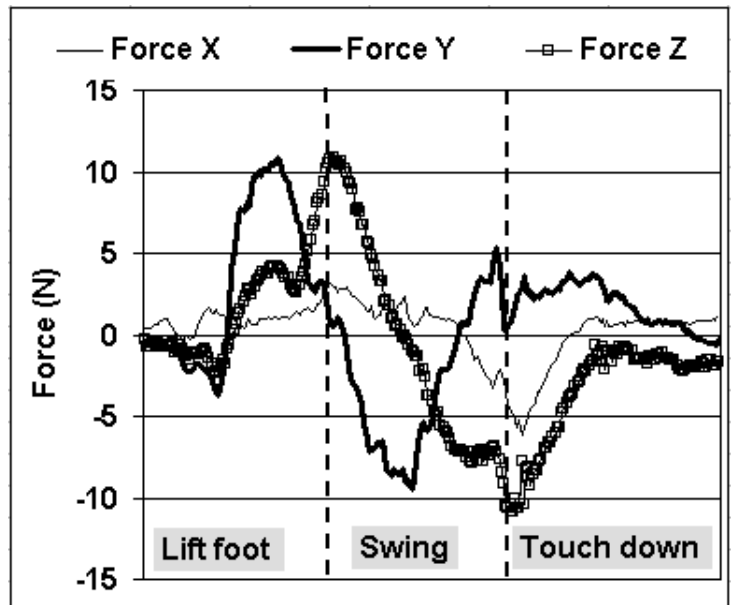

Fig. 7. Free mode forces during swing for larger $K_{t}$. (C) Rutgers University and UMDNJ. Reprinted by permission.

\subsection{Haptic rendering for walking over virtual terrain}

The Mobility Simulator transforms the input position, force and functioning mode of each RMA platform into haptic feedback to the feet and visual update of the virtual scene using the virtual surface specifications. The virtual ground model is stored on the graphics workstation, as a polygonal mesh with areas characterized by a haptic material. The haptic output data consists of two sets of values specifying the functioning mode to be used by each platform, the 6DOF forces and the vibrations to be applied to the user's foot.

\subsubsection{Haptic Material}

The ground surface is specified as a polygonal mesh that matches the shape of the visual 3D geometries in the virtual environment. The physical properties of the surface are specified using haptic materials, which are applied in layers that can be either distinct or mixed. The polygonal surface is unbreakable and the haptic materials can be placed on top of it in layers. This approach insures that the foot stepping down will always be supported if it penetrates through all the materials stacked on the surface. 
A haptic material is defined as a collection of numerical parameters describing the physical properties of the surface. Given the requirements of an interactive virtual reality simulation, the haptic modeling computations need to be efficient. Thus, the model is not intended to be physically accurate but rather a good approximation of reality. Hence, the set of variables used is limited to stiffness, damping, friction, haptic texture, and breaking coefficient.

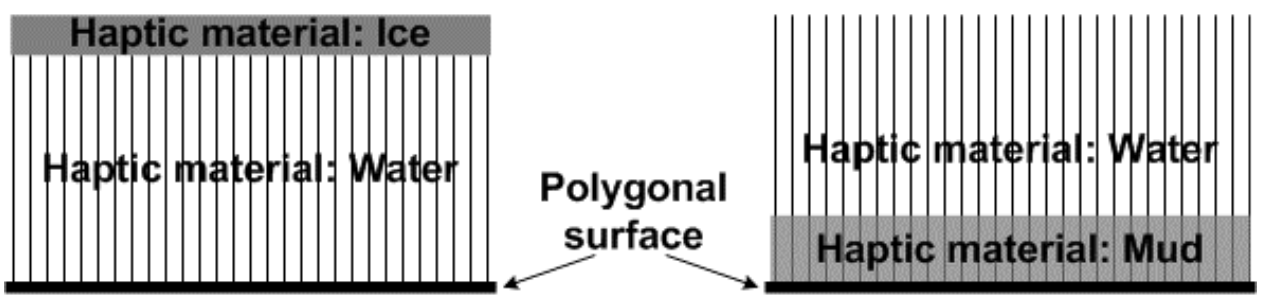

(a)

(b)

Fig. 8. Layered haptic materials: (a) distinct layers; (b) mixed layers.

The stiffness and damping coefficients are used for defining the material based on Hooke's law. Stepping on elastic materials is very common in every day life, however, the stiffness coefficient can be used to simulate Archimedes's law if considering the foot section constant when stepping into a liquid. The damping coefficient can be used to simulate the slow sinking sensation of walking on a thick carpet. A low friction coefficient can be used for simulating ice. If the foot applies horizontal forces to the material larger than the friction coefficient, the contact will break.

The haptic texture is defined as a vibration with a given amplitude and frequency. The breaking coefficient multiplied with the thickness of the material specifies the maximum force that the material can support. A haptic material is not rendered if the applied force is larger than its breaking force. The breaking coefficient makes it easy to simulate stepping on a thin layer of ice on top of a puddle of water.

4.2.2 Motion Rendering Stages

The processing necessary to calculate the graphics and haptic feedback can be divided into several stages that are executed at every simulation cycle (Figure 9). Only the swinging foot (free motion) is considered for the entire rendering process. The fixed foot (load compensation mode) is addressed only in the last stage of the process.

The process starts by reading the feet positions and functioning modes from the control interface. The functioning mode value is used to decide whether a foot should be moved or not. A foot in load compensation mode is kept fixed although the platform slides it backward.

The next stage requires the calculation of the change in real foot position to be added to the virtual feet. Because the simulator's workspace cannot cover the entire range of motion of the legs, it was necessary to scale the change in each foot's position to increase the virtual walking velocity so that the simulation felt real. The scale is also applied to the vertical displacement making it possible to negotiate realistic virtual obstacles that are visually larger than the platform work envelope.

The next phase updates the positions of the virtual feet with the calculated change. The changes are applied in a frame of reference aligned with the virtual avatar's walking direction calculated in the previous cycle. 


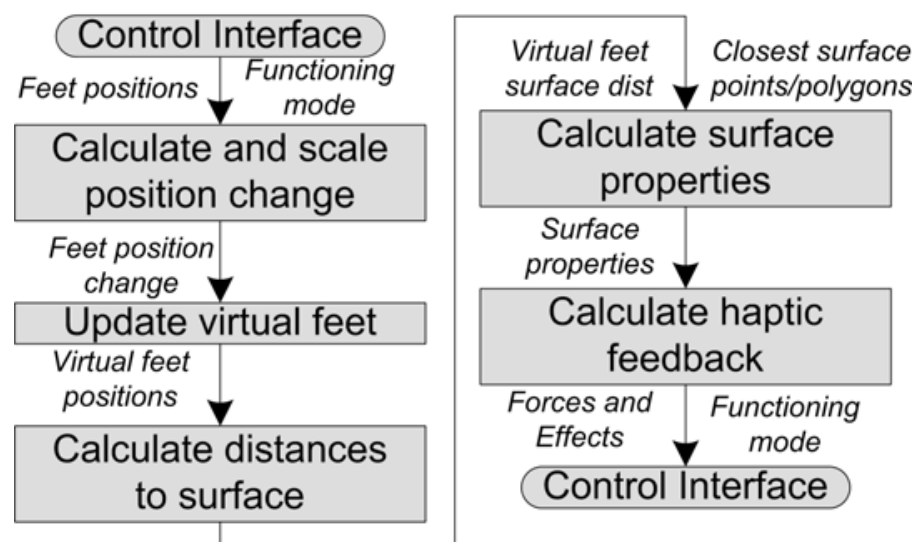

Fig. 8. Haptic rendering stages (C Rutgers University and UMDNJ. Reprinted by permission. After the feet have been mapped into the virtual world, the viewpoint has to be moved accordingly. Positioning the virtual camera above the center of the segment defined by the two feet yields good results although it is not what really happens with the center of gravity of a walking person.

The next stage is calculating the distance between the foot and the surface. This stage will also find the closest surface points to the foot and their corresponding polygons. Similar to the real case, certain surface properties are manifested above the surface (e.g. mud or snow) hence the distance to the surface is relevant to the haptic feedback even if there is no collision with the underlying ground. Based on the results of the previous stage the simulator can find the surface properties around the closest points on the surface.

The last stage of the process is the calculation of forces and haptic effects to be applied to the user's foot, based on the surface properties and the depth of the collision.

\subsubsection{Virtual Foot Modeling}

The interaction between the virtual foot and the virtual ground surface is based on the haptic mesh concept developed by Popescu (Popescu et al., 1999) as an extension to Ho's simpler haptic point concept (Ho et al., 1997). The virtual foot implemented for the Mobility Simulator is modeled as a mesh of points positioned on the shoe sole. From a haptic point of view, the RMA platforms can only render forces in one point. The use of a mesh of points to calculate the interaction of the foot with the surface is necessary for realistic surface contact calculation.

The number of points in the mesh should be minimized because it is directly proportional to the amount of collision detection calculations, and it increases factorially the number of contact stability calculations. The minimum number of mesh points has been determined to be five. One point is positioned in the center of the mesh, while the rest are positioned on a rectangle around it. The dimensions of the rectangle match the shape of the end-effector foot attachment plate to which the user's foot is secured.

\subsubsection{Ground Contact Evaluation}

When a foot touches the haptic surface the swinging phase of the foot is over and the support phase is about to begin. The switch between these two phases is tightly connected 
to the functioning of the servo-controller, which has to be notified to start sliding the support foot backward.

The foot/surface interaction is evaluated by calculating the forces applied by the surface (through the layers of haptic materials) to each of the points in the virtual foot mesh. The vertical components of these forces are then compared with the vertical components of the forces applied by the user's foot to the simulator. The latter forces are calculated by transforming the output of the force sensor mounted on top of the RMA platforms to each point in the mesh. A point of the foot haptic mesh is "supported" if the resultant of these vertical components is pointing upward.

The three contact possibilities that can be differentiated based on the "supported" status of the haptic mesh points are presented in Table 2 and Figure 10.

\begin{tabular}{|l|l|}
\hline Contact Status & Description \\
\hline No contact & None of the mesh points are supported \\
\hline Stable & Minimum three non-collinear points are supported \\
\hline Unstable & Remaining cases \\
\hline
\end{tabular}

Table 2. Contact status based on the haptic mesh point support.

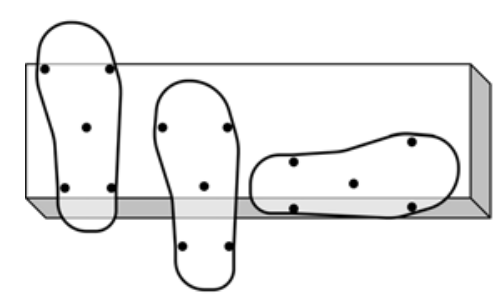

(a)

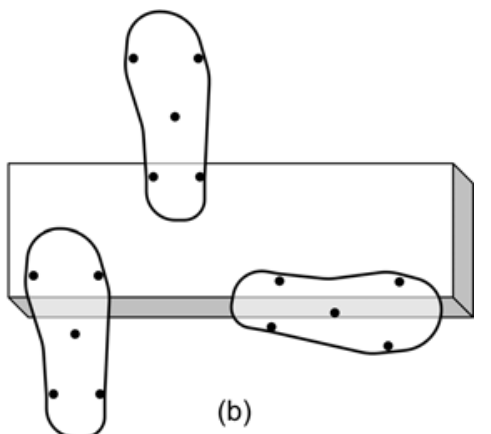

(b)

Fig. 10. Foot/surface contact types: (a) stable, (b) unstable. (c) Rutgers University and UMDNJ. Reprinted by permission.

The resultant of the haptic point forces is transmitted to the control interface to be applied to the swinging foot. If the swinging foot made a stable contact with the surface, the controller is notified to switch the functioning mode. The friction and surface textures are sent to the controller for both feet, regardless of their state.

\subsubsection{Low-level Haptic Effects}

The mobility simulator system is designed to execute most of the haptic calculations on the workstation, and render the results on the Stewart platforms using a reduced set of basic level effects:

- Apply a 6DOF force

- Change in position (positional jolt)

- Vibrations

These low level haptic effects are extensions of the haptic effects developed for a similar system using smaller Stewart platform robots, and designed for stroke rehabilitation in sitting (Boian et al., 2004a). 
The simulation can request the controller to apply a certain force during both free or foot support functioning modes. If the request is made while in free motion mode, the given forces are scaled using positive values of the free mode gains and then applied. The scaling is necessary to counteract the same damping behavior mentioned above. If a force request is made during the foot support mode, the given forces become essentially $\tau_{\text {des }}$ in Figure 3.

The changes in position are used as an alternative force requests during foot support mode. For instance, to simulate slipping on the ice, a lateral displacement is used instead of applying a lateral force. This is preferred because a known displacement is more controllable and can be adjusted to a comfortable level easier than applying a force which will have a different effect from a user to another, mainly due to differences in weight.

The vibrations are used to simulate surface textures. The frequency and amplitude of the vibrations are calculated on the graphical workstation and sent to the controller. The vibrations are rendered only as changes in orientation around the Y-axis (back-front direction) because it interferes the least with the rest of the foot measurements necessary to calculate the direction of motion, or intersection with the virtual surface.

\section{Acknowledgment}

The research reported here was supported by grants from the National Science Foundation (BES 0201687 and BES 9708020) and from the New Jersey Commission for Science and Technology.

\section{References}

Boian, R.F.; Deutsch, J.E.; Lee, C.S.; G.C., Burdea \& Lewis J.A. (2003). Haptic Effects for Virtual Reality-based Post-Stroke Rehabilitation. Proceedings of the Eleventh Symposium on Haptic Interfaces For Virtual Environment And Teleoperator Systems, pp. 247-253, Los Angeles, CA, March 2003.

Boian, R.F.; Bouzit, M.; Burdea, G.C. \& Deutsch, J.E. (2004a). Dual Stewart Platform Mobility Simulator. Proceedings of IEEE EMBS 2004, September 2004

Boian, R.F.; Burdea, G.C.; Deutsch, J.E. \& Winter, S. H. (2004b). Street Crossing Using a Virtual Environment Mobility Simulator. Proceedings of IWVR 2004, pp. 27-33, Lausanne, Switzerland, 2004.

Boian, R.F. (2005). Robotic Mobility Rehabilitation System Using Virtual Reality. Electrical and Computer Engineering, Rutgers University, January 2005.

Bouguilla, L. \& Sato, M. (2002). Virtual locomotion system for large-scale virtual environment. Proceedings of the IEEE Virtual Reality Conference, pp. 291-292, March 2002.

Burdea, G. (1996). Force and Touch Feedback for Virtual Reality. John Wiley \& Sons, New York, NY, USA, 1996.

Christensen, R.R. ; Hollerbach, J.M.; Xu, Y. \& Meek, S.G. (2000). Inertial-force feedback for the treadport locomotion interface. Presence, vol. 9, no. 1, pp.1-14, February 2000.

Dietz, V.; Wirz, M.; Curt, A. \& Colombo, G. (1998). Locomotor pattern in paraplegic patients: training effects and recovery of spinal cord function. Spinal Cord 1998; 36: pp. 380-390.

Ho, C.; Basdogan, C. \& Srinivasan, M. (1997). Haptic Rendering: Point- and Ray-Based Interactions. Proceedings of the Second PHANToM Users Group Workshop, October 2007, Dedham, MA. 
Hollerbach, J.M. (1999). Locomotion interfaces. In: Handbook of Virtual Environments Technology, Stanney, K.M., (Ed.), pp. 239-254, Lawrence Erlbaum Associates, Inc., 2002.

Iwata, H. (1999). Walking about virtual environments on infinite floor. Proceedings of IEEE Virtual Reality'99, pp. 286-293, 1999, Houston, TX.

Iwata, H. \& Yoshida, Y. (1999). Path reproduction tests using a torus treadmill. Presence: Teleoperators and Virtual Environments, vol. 8, pp. 587-597, 1999.

Iwata, H.; Yano, H. \& Nakaizumi F. (2001). Gaitmaster: A versatile locomotion interface for uneven virtual terrain. Proceedings of the IEEE VR2001 Conference, pp. 131-137, 2001.

Jorgensen, H.S. ; Nakayama, H.; Raaschou, \& Olsen, T.S. (1995). Recovery in walkingcfunction in stroke patients: The copenhagen stroke study. Archives of Physical Medicine and rehabilitation, vol. 76, no. 1, pp. 27-32, 1995.

Miyasato T. (2000). Tele-nursing system with realistic sensations using virtual locomotion interface. Proceedings of the 6th ERCIM Workshop User Interfaces for All, October 2000, Florence, Italy.

Nicol, D.J.; Granat, M.H.; Baxendale, R.H. \& Tuson, S.J. (1995). Evidence for a human spinal stepping generator. Brain Res 1995; 684: pp. 230-232.

Noma, H.; Sugihara, T. \& Miyasato T. (2000). Development of Ground Surface Simulator for Trl-E-Merge System. Proceedings of IEEE Virtual Reality 2000, pp. 217-224, March 2000.

Parsons, J.; Lampton, D.R.; Parsons, K.A.; Knerr, B.W.; Russell, D.; Martin, G.; Daly, J.; Kline, B. \& Weaver. M. (1998) Fully immersive team training: A networked testbed for ground based training missions. Proceedings of Interservice/Industry Training, Simulation and Education Conference, Orlando, FL, 1998.

Popescu, V.; Burdea, G. \& Bouzit, M. (1999). Virtual Reality Simulation Modeling for a Haptic Glove. Computer Animation'99, pp. 195-200, May 1999, Geneva, Switzerland.

Riva, G. (2000). Virtual Reality In Rehabilitation Of Spinal Cord Injuries: A Case Report. Rehabilitation Psychology, 45 (1), pp. 1-8, 2000

Sveistrup, H. (2004). Motor rehabilitation using virtual reality, Journal of NeuroEngineering and Rehabilitation 2004.

Templeman, J.N.; Denbrook, P.S. \& Sibert. L.E. (1999). Maintaining spatial orientation during travel in an immersive virtual environment. Presence: Teleoperators and Virtual Environments, Vol 8, pp. 598-617, 1999.

Wandel, A.; Jorgensen, H.S.; Nakayama, H.; Raaschou, H.o. \& Olsen, T.S. (2000). Prediction of walking function in stroke patients with initial lower extremity paralysis: the copenhagen stroke. Archives of Physical Medicine and rehabilitation, Vol. 81, No. 6, pp. 736-738, 2000.

Wang, Z.; Bauernfeind, K. \& Sugar. T. (2003). Omni-direactional treadmill system. Proceedings of the Symposium on Haptic Interfaces for Virtual Environment and Teleoperator Systems, pp. 367-373, Los Angeles, CA, March 2003. 


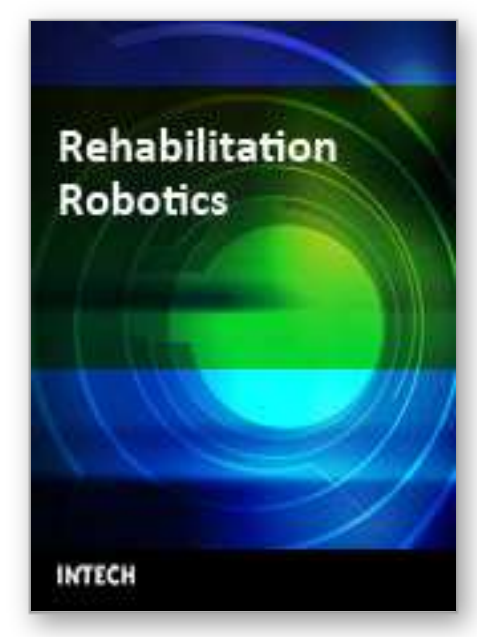

\author{
Rehabilitation Robotics \\ Edited by Sashi S Kommu
}

ISBN 978-3-902613-04-2

Hard cover, 648 pages

Publisher I-Tech Education and Publishing

Published online 01, August, 2007

Published in print edition August, 2007

The coupling of several areas of the medical field with recent advances in robotic systems has seen a paradigm shift in our approach to selected sectors of medical care, especially over the last decade.

Rehabilitation medicine is one such area. The development of advanced robotic systems has ushered with it an exponential number of trials and experiments aimed at optimising restoration of quality of life to those who are physically debilitated. Despite these developments, there remains a paucity in the presentation of these advances in the form of a comprehensive tool. This book was written to present the most recent advances in rehabilitation robotics known to date from the perspective of some of the leading experts in the field and presents an interesting array of developments put into 33 comprehensive chapters. The chapters are presented in a way that the reader will get a seamless impression of the current concepts of optimal modes of both experimental and ap- plicable roles of robotic devices.

\title{
How to reference
}

In order to correctly reference this scholarly work, feel free to copy and paste the following:

Rares F. Boian and Grigore C. Burdea \& Judith E. Deutsch (2007). Robotics and Virtual Reality Applications in Mobility Rehabilitation, Rehabilitation Robotics, Sashi S Kommu (Ed.), ISBN: 978-3-902613-04-2, InTech, Available from:

http://www.intechopen.com/books/rehabilitation_robotics/robotics_and_virtual_reality_applications_in_mobility_ rehabilitation

\section{INTECH}

open science | open minds

\author{
InTech Europe \\ University Campus STeP Ri \\ Slavka Krautzeka 83/A \\ 51000 Rijeka, Croatia \\ Phone: +385 (51) 770447 \\ Fax: +385 (51) 686166 \\ www.intechopen.com
}

\author{
InTech China \\ Unit 405, Office Block, Hotel Equatorial Shanghai \\ No.65, Yan An Road (West), Shanghai, 200040, China \\ 中国上海市延安西路65号上海国际贵都大饭店办公楼405单元 \\ Phone: +86-21-62489820 \\ Fax: $+86-21-62489821$
}


(C) 2007 The Author(s). Licensee IntechOpen. This chapter is distributed under the terms of the Creative Commons Attribution-NonCommercial-ShareAlike-3.0 License, which permits use, distribution and reproduction for non-commercial purposes, provided the original is properly cited and derivative works building on this content are distributed under the same license. 